\title{
MONATSSCHRIFT \\ FÜR \\ PSYCHIATRIE UND NEUROLOGIE
}

BEGRÜNDET VON C. WERNICKE UND TH. ZIEHEN

UNTER MITWIRKUNG VON

\begin{tabular}{|c|c|c|c|}
\hline \multirow[t]{3}{*}{$\begin{array}{l}\text { K. KLEIST } \\
\text { FRANKFÜRT A. M. }\end{array}$} & $\begin{array}{c}\text { 0. PÖTZL } \\
\text { PRAG }\end{array}$ & $\underset{\text { WIEN }}{\text { E. REDLICH }}$ & $\begin{array}{l}\text { P. SCHRÖDER } \\
\text { LEIPZIG }\end{array}$ \\
\hline & HER & EGEBEN VON & \\
\hline & K. I & $\begin{array}{l}\text { HOEFFER } \\
\text { ERLIN }\end{array}$ & \\
\hline
\end{tabular}

Bd. LXIII

.Mit zahlreichen Abbildungen im Text tuuUetoerf?a-fel

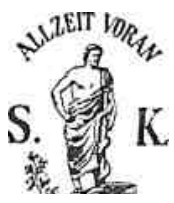


Alle Rechte vorbehalten.

Weimar. — Druck von R. Wagner Sohn. 



\section{Inhalts -Verzeichnis.}

Originalarbeiten.

e

Birnbaum, K., Persönlichkeit und Psychose .......................346

Bonhoeffer, K., Rückblick auf die Geschichte der Berliner Gesellschaft für Psychiatrie und Nervenkrankheiten . 289

Bratz, E., Fürsorge für Nervöse und Seelisch-Abnorme. Ein Rückblick und Ausblick , . . •................... 35g

Büchler, P., Wie das Cholesterin in die Zerebrospinalflüssig-

keit gelangt

Fischer, M. H., Über die spontane Abweichreaktion. Be merkungen zur gleichnamigen Mitteilung von $H$. Hoff und P. Schilder (Bd. 62, S. 134).....................................282 Fünfgeld, E., Über die pathologische Anatomie der Schizophrenie und ihre Bedeutung für die Abtrennung ,aty pischer" periodisch verlaufender Perioden 1

Hildebrandt, K., Wertbegriffe in der Psychiatrie 373

Hirsch, E., Pathologische Schlafzustähde bei Herderkrangungen des Mittelhirns

Jossmann, P., Die Beziehungen der motorischen Amusie zu

den apraktischen Störungen

Kapp, F., Über zwei Fälle von Nystagmus rotatorius bei

Stirnhirnschädigung 130

Münzer, F. Th., Zur Frage der symptomatischen Narko

lepsie nach Enzephalitis lethargica

97

Neisser, C., Die Weiterentwicklung der praktischen

Psychiatrie,

insbesondere der Anstaltstherap^ ${ }^{\wedge}$ Em Sinne Griesingers 314

PohlWm, K., Die pathogenetische Bedeutung der Gelegenheits ursachen für das Delirium tremens.

69

—, - Z Zur Pathogenese der akuten Halluzinose der Trinker.

82

—, - , Die Persönlichkeit und das Milieu Delirium-tremens-

Kranker der Charite aus den Jahren 1912 - 1925

136 


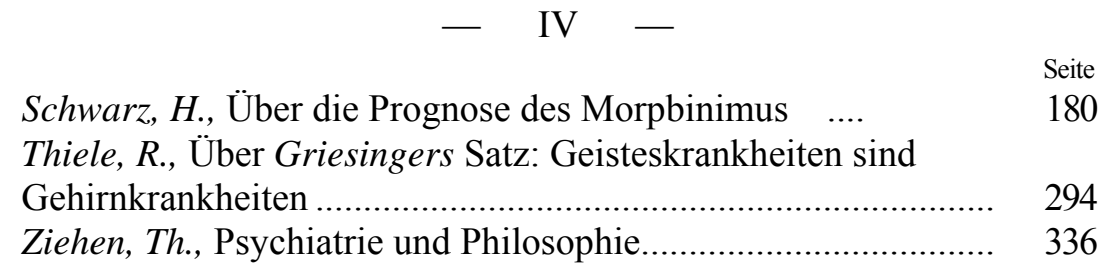

Deutscher Verein für Psychiatrie

Buchbesprechungen .

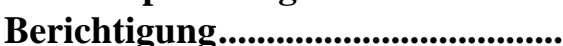


288

in, 286

112 